\title{
SOBOLEV REGULARITY FOR MONGE-AMPÈRE TYPE EQUATIONS
}

\author{
GUIDO DE PHILIPPIS AND ALESSIO FIGALLI
}

\begin{abstract}
In this note we prove that, if the cost function satisfies some necessary structural conditions and the densities are bounded away from zero and infinity, then strictly c-convex potentials arising in optimal transportation belong to $W_{\text {loc }}^{2,1+\kappa}$ for some $\kappa>0$. This generalizes some recents results [9, 10, 22] concerning the regularity of strictly convex Alexandrov solutions of the Monge-Ampère equation with right hand side bounded away from zero and infinity.
\end{abstract}

\section{INTRODUCTION}

Let $\Omega \subset \mathbb{R}^{n}$ be a bounded open set. We want to investigate the regularity of solutions to Monge-Ampère type equations of the form

$$
\operatorname{det}\left(D^{2} u-\mathcal{A}(x, D u)\right)=f \quad \text { in } \Omega,
$$

where $f \geq 0$ and $\mathcal{A}(x, p)$ is a $n \times n$ symmetric matrix.

This class of equations naturally arises in optimal transportation, and in reflector and refractor shape design problems. In these applications, the matrix $\mathcal{A}$ and the right and side $f$ are given by

$$
\mathcal{A}(x, D u(x))=-D_{x x} c\left(x, T_{u}(x)\right), \quad f(x)=\left|\operatorname{det}\left(D_{x y} c\left(x, T_{u}(x)\right)\right)\right| \frac{\rho_{0}(x)}{\rho_{1}\left(T_{u}(x)\right)},
$$

where $c(x, y)$ represents the cost function, $\rho_{0}$ and $\rho_{1}$ are probability densities, and $T_{u}$ is the optimal transport map sending $\rho_{0}$ onto $\rho_{1}$. Under a twist assumption on the cost (see (C2) below), the map $T_{u}$ is uniquely determined through the relation

$$
-D_{x} c\left(x, T_{u}(x)\right)=D u(x) .
$$

Moreover, when $\mathcal{A} \equiv 0$ the above equation reduces to the classical Monge-Ampère equation.

The regularity for the above class of equations has received a lot of attention in the last years [11, 12, 17, 18, 19, 21, 23, 24. In particular, under some necessary structural conditions on $\mathcal{A}$ (see (C1) below), one can show that if $f$ is smooth then $u$ is smooth as well [18, 21, 23, 24. In addition, it is proved in [11] that solutions are locally $C^{1, \alpha}$ when $f$ is merely bounded away from zero and infinity (see also [12, 17]).

Recently, the authors introduced new techniques to address the Sobolev regularity of $u$ when $\mathcal{A} \equiv 0$ : more precisely, under the assumption that $f$ is bounded away from zero and infinity, it is proved in [9] that $D^{2} u \in L \log L_{\text {loc }}(\Omega)$, and with a variant of the same techniques this result has been improved in [10] to $u \in W_{\text {loc }}^{2,1+\kappa}(\Omega)$ for some $\kappa>0$ (see also [22]). Let us mention that these results played a crucial role in [1, 2] to show the existence of distributional solutions to the semi-geostrophic system. 
The aim of this paper is to extend the $W_{\text {loc }}^{2,1+\kappa}$ regularity to the general class of Monge-Ampère equations in (1.1). Apart from its own interest, it seems likely that this result could have applications in the study of generalized semi-geostrophic system on Riemannian manifolds [6], in particular on the sphere [20] and its perturbations [7, 8, 13, 14].

In order to describe our result, we need to introduce some more notation and the main assumptions on the cost functions.

Let $X \subset \mathbb{R}^{n}$ be an open set, and $u: X \rightarrow \mathbb{R}$ be a $c$-convex function, i.e., $u$ can be written as

$$
u(x)=\max _{y \in \bar{Y}}\left\{-c(x, y)+\lambda_{y}\right\}
$$

for some open set $Y \subset \mathbb{R}^{n}$, and $\lambda_{y} \in \mathbb{R}$ for all $y \in \bar{Y}$. We are going to assume that $u$ is an Alexandrov solution of (1.1) inside some open set $\Omega \subset X$, i.e.,

$$
\left|\partial^{c} u(E)\right|=\int_{E} f \quad \text { for all } E \subset \Omega \text { Borel, }
$$

where

$$
\partial^{c} u(E):=\bigcup_{x \in E} \partial^{c} u(x), \quad \partial^{c} u(x):=\left\{y \in \bar{Y}: u(x)=-c(x, y)+\lambda_{y}\right\}
$$

and $|F|$ denotes the Lebesgue measure of a set $F$. It is well-known that, in order to prove some regularity results, (1.1) needs to be coupled with some boundary conditions: for instance, when $\mathcal{A} \equiv 0$ and $f \equiv 1$, solutions are smooth whenever they are strictly convex, and to obtain strict convexity some suitable boundary conditions are needed [3, 5 .

For the general case in (1.1), let $u$ be a $c$-convex function associated to an optimal transport problem, and for any $y \in \bar{Y}$ define the contact set

$$
\Lambda_{y}:=\left\{x \in X: u(x)=-c(x, y)+\lambda_{y}\right\} .
$$

Under some structural assumptions on the cost functions (which we shall describe below) and some convexity hypotheses on the supports of the source and target measure, it has been proved in 11] that $u$ is an Alexandrov solution of (1.1) inside $X$, and it is strictly c-convex (i.e., for any $y \in \partial^{c} u(X)$ the contact set $\Lambda_{y}$ reduces to one point) provided $f$ is bounded away from zero and infinity.

Here, since we want to investigate the interior regularity of $u$, instead of assuming that $u$ comes from an optimal transportation problem where the supports of the source and target measure enjoy some global " $c$-convexity" property, we work assuming directly that $u$ is a strictly $c$-convex Alexandrov solution near some point $\bar{x} \in X$, and we prove regularity of $u$ in a neighborhood of $\bar{x}$. This has the advantage of making our result more general and flexible for possible future applications.

Hence, we assume that there exist $(\bar{x}, \bar{y}) \in X \times Y$ such that $\Lambda_{\bar{y}}=\{\bar{x}\}$, we consider a neighborhood $\Omega$ of $\bar{x}$ given by

$$
\Omega:=\left\{x \in X: u(z)<-c(x, \bar{y})+\lambda_{y}+\delta\right\},
$$

where $\delta>0$ is a small constant chosen so that $\Omega \subset \subset X$ and $\partial^{c} u(\Omega) \subset \subset Y$ (such a constant $\delta$ exists because $\Lambda_{\bar{y}}:=\{\bar{x}\}$ ). Also, we assume that $u$ is an Alexandrov solution of

$$
\begin{cases}\operatorname{det}\left(D^{2} u-\mathcal{A}(x, D u)\right)=f & \text { in } \Omega, \\ u=-c(\cdot, \bar{y})+\text { const } & \text { on } \partial \Omega .\end{cases}
$$


Before stating our result, let us introduce the main conditions on the cost function: let $\Omega$ be as above, and let $\mathcal{O} \subset \subset Y$ be a open neighborhood of $\partial^{c} u(\Omega)$. We define

$$
\|c\|:=\|c\|_{C^{3}(\bar{\Omega} \times \overline{\mathcal{O}})}+\left\|D_{x x y y} c\right\|_{L^{\infty}(\bar{\Omega} \times \overline{\mathcal{O}})}+\left\|\log \left|\operatorname{det} D_{x y} c\right|\right\|_{L^{\infty}(\bar{\Omega} \times \overline{\mathcal{O}})}
$$

and assume that the following hold:

(C0) $\|c\|<\infty$.

(C1) For every $x \in \Omega$ and $p:=-D_{x} c(x, y)$ with $y \in \mathcal{O}$, it holds

$$
D_{p_{k} p_{\ell}} \mathcal{A}_{i j}(x, p) \xi_{i} \xi_{j} \eta_{k} \eta_{\ell} \geq 0, \quad \forall \xi, \eta \in \mathbb{R}^{n}, \xi \cdot \eta=0,
$$

where $\mathcal{A}$ is defined through $c$ by $\mathcal{A}_{i j}(x, p):=-D_{x_{i} x_{j}} c(x, y)$, and we use the summation convention over repeated indices.

Let us point out that, up to reduce the size of $\Omega$ and $\mathcal{O}$ (this is possible because $\Omega \rightarrow\{\bar{x}\}$ and $\partial^{c} u(\Omega) \rightarrow\{\bar{y}\}$ as $\delta \rightarrow 0$ ), as a consequence of (C0) (more precisely, from the fact that $\operatorname{det} D_{x y} c(\bar{x}, \bar{y}) \neq 0$ and by the implicit function theorem) we can assume that the following holds:

(C2) For every $(x, y) \in \Omega \times \mathcal{O}$, the maps $x \in \Omega \mapsto-D_{y} c(x, y)$ and $y \in \mathcal{O} \mapsto-D_{x} c(x, y)$ are diffeomorphisms on their respective ranges.

We also notice that, because of the boundary condition $u=-c(\cdot, \bar{y})+$ const on $\partial \Omega$, if $f$ is bounded away from zero and infinity inside $\Omega$, then any $c$-convex Alexandrox solution of (1.4) is strictly $c$-convex inside $\Omega$ (this is an immediate consequence of [11, Remark 7.2]). Here is our result:

Theorem 1.1. Let $u: \Omega \rightarrow \mathbb{R}$ be a c-convex Alexandrov solution of (1.4). Assume that $c$ satisfies conditions (C0)-(C2), and that $0<\lambda \leq f \leq 1 / \lambda$. Then $u \in W_{\mathrm{loc}}^{2,1+\kappa}(\Omega)$ for some $\kappa>0$.

Theorem 1.1 generalizes the corresponding result for the classical Monge-Ampère equation to the wider class of equations considered here. With respect to the arguments in [9, 10], additional complications arise from the fact that, in contrast with the classical Monge-Ampère equation, in general (1.1) is not affinely invariant.

Acknowledgements: AF is partially supported by NSF Grant DMS-0969962. Both authors acknowledge the support of the ERC ADG Grant GeMeThNES. The first author thanks the hospitality of the Mathematics Department at the University of Texas at Austin, where part of this work has been done.

\section{NotATION AND PRELIMINARY RESUlts}

Through all the paper, we call universal any constant which depends only on the data, i.e., on $n$, $\Omega, \mathcal{O}, \lambda$, and $\|c\|$. We use $C$ to denote a universal constant larger than 1 whose value may change from line to line, and we use the notation $a \approx b$ to indicate that the ratio $a / b$ is bounded from above and below by positive universal constants.

An immediate consequence of the definition of $c$-convexity (1.2) is that, for any $x_{0} \in X$, there exists $y_{0} \in \bar{Y}$ such that

$$
u(x) \geq-c\left(x, y_{0}\right)+u\left(x_{0}\right)+c\left(x_{0}, y_{0}\right) \quad \forall x \in X,
$$

and in this case $y_{0} \in \partial^{c} u\left(x_{0}\right)$. If in addition $u \in C^{2}$, then it is easily seen that $D u\left(x_{0}\right)=$ $-D_{x} c\left(x_{0}, y_{0}\right)$ and $D^{2} u\left(x_{0}\right) \geq-D_{x x} c\left(x_{0}, y_{0}\right)=\mathcal{A}\left(x_{0}, D u\left(x_{0}\right)\right)$, where $\mathcal{A}$ is defined in (C1) above. In particular equation (1.4) is degenerate elliptic when restricted to $c$-convex function. 
It has been discovered independently in [11] and [17 that, because of (C1), for any $x_{0} \in \Omega$ and $y_{0} \in \partial^{c} u\left(x_{0}\right)$, through the change of variables $x \mapsto q(x):=-D_{y} c\left(x, y_{0}\right)$ the function

$$
\bar{u}(q):=u(x(q))+c\left(x(q), y_{0}\right)-u\left(x_{0}\right)-c\left(x_{0}, y_{0}\right)
$$

has convex level sets inside $\Omega$ (here and in the sequel $x(q)$ denotes the inverse of $q(x)$, which is well defined because of (C2)). Moreover $\bar{u}$ is $\bar{c}$-convex, where

$$
\bar{c}(q, y):=c(x(q), y)-c\left(x(q), y_{0}\right),
$$

see [11, Theorem 4.3].

Since $u$ solves (1.4) one can check by a direct computation that $\bar{u}$ solves

$$
\operatorname{det}\left(D^{2} \bar{u}-\mathcal{B}(q, D \bar{u})\right)=g \text {, }
$$

with

$$
\mathcal{B}_{i j}(q, p)=-D_{q_{i} q_{j}} \bar{c}\left(q, T_{\bar{u}}(q)\right) \quad \text { and } \quad g(q)=f(x(q))\left[\operatorname{det} D_{x y} c\left(x(q), T_{\bar{u}}(q)\right)\right]^{-2},
$$

where $T_{\bar{u}}$ is the map uniquely identified by the relation $D \bar{u}(q)=-D_{q} c\left(q, T_{\bar{u}}(q)\right)$. Moreover it holds

$$
\mathcal{B}_{i j}(\cdot, 0) \equiv 0, \quad D_{p} \mathcal{B}_{i j}(\cdot, 0) \equiv 0,
$$

so using Taylor's formula we can write

$$
\mathcal{B}_{i j}(q, D \bar{u})=\mathcal{B}_{i j, k \ell}(q, D \bar{u}) \partial_{k} \bar{u} \partial_{l} \bar{u}
$$

where

$$
\mathcal{B}_{i j, k \ell}(q, D \bar{u}(q)):=\int_{0}^{1} D_{p_{k} p_{\ell}} \mathcal{B}_{i j}(q, \tau D \bar{u}(q)) d \tau .
$$

In addition, since condition (C1) is tensorial [21, 19, 16] and $\|c\|$ involves only mixed fourth derivative, it is easily seen that $\|\bar{c}\| \approx\|c\|$ and $\mathcal{B}$ satisfies the same assumptions as $\mathcal{A}$. In particular (C1) and (2.7) imply that

$$
\mathcal{B}_{i j, k \ell} \xi_{i} \xi_{j} \eta_{k} \eta_{\ell} \geq 0 \quad \forall \xi \cdot \eta=0
$$

Given a $C^{1} c$-convex function as above, for any $x_{0} \in \Omega, y_{0}=T_{u}\left(x_{0}\right)$, and $h \in \mathbb{R}^{+}$, we define the section centered at $x_{0}$ of height $h$ as

$$
S_{h}^{u}\left(x_{0}\right):=\left\{x \in \Omega: u(x) \leq-c\left(x, y_{0}\right)+u\left(x_{0}\right)+c\left(x_{0}, y_{0}\right)+h\right\} .
$$

Assuming that $S_{h}^{u}\left(x_{0}\right) \subset \Omega$, through the change of variables $x \mapsto q(x):=-D_{y} c\left(x, y_{0}\right)$ this section is transformed into the convex set

$$
Q_{h}^{\bar{u}}\left(q_{0}\right):=-D_{y} c\left(S_{h}^{u}\left(x_{0}\right), y_{0}\right)=\{q: \bar{u}(q) \leq h\} .
$$

When no confusion arises, we will often abbreviate $S_{h}\left(x_{0}\right)$ and $Q_{h}\left(q_{0}\right)$ for $S_{h}^{u}\left(x_{0}\right)$ and $Q_{h}^{\bar{u}}\left(q_{0}\right)$.

We also recall [15] that, given an open bounded convex set $Q$, there exists an ellipsoid $E$ such that

$$
E \subset Q \subset n E,
$$

where the dilation is done with respect to the center of $E$. We refer to it as the John ellipsoid of $Q$, and we say that $Q$ is normalized if $E=B(0,1)$. An immediate consequence of (2.9) is that any open bounded convex set $Q$ admits an affine transformation $L$ such that $L(Q)$ is normalized. 
Hence, given $u$ and $S_{h}^{u}\left(x_{0}\right)$ as above, we can consider $\bar{u}$, his section $Q_{h}^{\bar{u}}\left(q_{0}\right)$, and the normalizing affine transformation $L$. Then we define $\bar{w}: L\left(Q_{h}\right) \rightarrow \mathbb{R}$ as

$$
\bar{w}\left(q^{\prime}\right):=(\operatorname{det} L)^{2 / n} \bar{u}(q), \quad q^{\prime}:=L q .
$$

It is easy to check that $\bar{w}$ solves

$$
\operatorname{det}\left(D^{2} \bar{w}\left(q^{\prime}\right)-\mathcal{C}\left(q^{\prime}, D \bar{w}\left(q^{\prime}\right)\right)\right)=g\left(L^{-1} q^{\prime}\right),
$$

where

$$
\mathcal{C}\left(q^{\prime}, D \bar{w}\left(q^{\prime}\right)\right):=(\operatorname{det} L)^{2 / n}\left(L^{*}\right)^{-1} \mathcal{B}\left(L^{-1} q^{\prime},(\operatorname{det} L)^{-2 / n} L^{*} D \bar{w}\left(q^{\prime}\right)\right) L^{-1}
$$

Up to an isometry, we can assume that

$$
E=\left\{q: \sum_{i=1}^{n} \frac{q_{i}^{2}}{r_{i}^{2}} \leq 1\right\},
$$

with $r_{1} \leq \ldots \leq r_{n}$. Then $L^{-1}=\operatorname{diag}\left(r_{1}, \ldots, r_{n}\right)$, and

$$
\mathcal{C}_{i j}\left(q^{\prime}, D \bar{w}\left(q^{\prime}\right)\right)=\mathcal{C}_{i j, k \ell}\left(q^{\prime}, D \bar{w}\left(q^{\prime}\right)\right) \partial_{k} \bar{w} \partial_{\ell} \bar{w}
$$

with

$$
\mathcal{C}_{i j, k \ell}\left(q^{\prime}, D \bar{w}\left(q^{\prime}\right)\right)=\left(r_{1} \ldots r_{n}\right)^{2 / n} \frac{r_{i} r_{j}}{r_{k} r_{\ell}} \mathcal{B}_{i j, k \ell}(q, D \bar{u}(q)),
$$

see (2.7). Moreover, by (2.8) (or again because of the tensorial nature of condition (C1))

$$
\mathcal{C}_{i j, k \ell} \xi_{i} \xi_{j} \eta_{k} \eta_{\ell} \geq 0 \quad \forall \xi \cdot \eta=0 .
$$

Still with the same notation as above, we also define the normalized size of a section $S_{h}\left(x_{0}\right)$ as

$$
\boldsymbol{\alpha}\left(S_{h}\left(x_{0}\right)\right)=\boldsymbol{\alpha}\left(Q_{h}\left(q_{0}\right)\right):=\frac{|L|^{2}}{(\operatorname{det} L)^{2 / n}} .
$$

Notice that, even if $L$ may not be unique, $\boldsymbol{\alpha}$ is well defined up to universal constants. In case $u$ is $C^{2}$ in a neighborhood of $x_{0}$, by a simple Taylor expansion of $\bar{u}$ around $q_{0}$ it is easy to see that there exists $h\left(x_{0}\right)>0$ small such that

$$
\boldsymbol{\alpha}\left(S_{h}\left(x_{0}\right)\right)=\boldsymbol{\alpha}\left(Q_{h}\left(q_{0}\right)\right) \approx\left|D^{2} \bar{u}\left(q_{0}\right)\right| \quad \forall h \leq h\left(x_{0}\right),
$$

where $q_{0}:=q\left(x_{0}\right)$. Since $u$ and $\bar{u}$ are related by a diffeomorphism, the following lemma holds:

Lemma 2.1. Let $\Omega^{\prime} \subset \Omega$, and $u \in C^{2}\left(\Omega^{\prime}\right)$ be a strictly c-convex function such that $\|D u\|_{L^{\infty}\left(\Omega^{\prime}\right)}$ is universally bounded. Then there exists a universal constants $M_{1}$ such that the following holds: For every $x_{0} \in \Omega$ there exists a height $\bar{h}\left(x_{0}\right)>0$ such that if $\left|D^{2} u\left(x_{0}\right)\right| \geq M_{1}$, then

$$
\left|D^{2} u\left(x_{0}\right)\right| \approx \boldsymbol{\alpha}\left(S_{h}\left(x_{0}\right)\right) \quad \forall h \leq \bar{h}\left(x_{0}\right) .
$$

Proof. Differentiating twice the relation (2.1) we obtain

$$
D_{q q} \bar{u}=D_{q} x D_{x x} u+D_{q q} x D_{x} u+D_{q} x D_{x x} c+D_{q q} x D_{x} c,
$$

which implies that

$$
\nu\left|D^{2} \bar{u}\left(q_{0}\right)\right|-C\left(1+\left|D u\left(x_{0}\right)\right|\right) \leq\left|D^{2} u\left(x_{0}\right)\right| \leq \frac{1}{\nu}\left|D^{2} \bar{u}\left(q_{0}\right)\right|+C\left(1+\left|D u\left(x_{0}\right)\right|\right)
$$

for some universal constants $\nu, C>0$. Since by assumption $D u$ is universally bounded inside $\Omega^{\prime}$, (2.17) follows by (2.18) and (2.16), provided $M_{1}$ is sufficiently large. 
We show now some geometric properties of sections and some estimates for solutions of (1.4) which will play a major role in the sequel. Here, the dilation of a section $S_{h}(x)$ is intended with respect to $x$.

Proposition 2.2 (Properties of section). Let $u$ be a c-convex Aleksandrov solution of (1.4) with $0<\lambda \leq f \leq 1 / \lambda$. Then, for any $\Omega^{\prime} \subset \subset \Omega^{\prime \prime} \subset \subset \Omega$, there exists a positive constant $\rho=\rho\left(\Omega^{\prime}, \Omega^{\prime \prime}\right)$ such that the following properties hold:

(i) $S_{h}^{u}(x) \subset \Omega^{\prime \prime}$ for any $x \in \Omega^{\prime}, 0 \leq h \leq 4 \rho$.

(ii) There exist $0<\alpha_{1}<\alpha_{2}$ universal such that for all $\mu \in(0,1)$

$$
\mu^{\alpha_{2}} S_{h}^{u}(x) \subset S_{\lambda h}^{u}(x) \subset \mu^{\alpha_{1}} S_{h}^{u}(x)
$$

for any $x \in \Omega^{\prime}, 0 \leq 2 h \leq \rho$.

(iii) There exists a universal constant $\sigma<1$ such that, if $S_{h}^{u}(x) \cap S_{h}^{u}(y) \neq \emptyset$, then $S_{h}^{u}(y) \subset$ $S_{h / \sigma}^{u}(x)$ for any $x, y \in \Omega^{\prime}, 0 \leq h \leq \sigma \rho$.

(iv) $\cap_{0<h \leq \rho} S_{h}^{u}(x)=\{x\}$.

Proof. Points (i) and (iv) follow from the strict $c$-convexity of $u$ shown in [11, section 7], and the fact that the modulus of strict $c$-convexity is universal (this last fact follows by a simple compactness argument in the spirit of [4, Theorem 1']).

Point (iii) corresponds the engulfing property of sections proved in [11, Theorem 9.3].

The second inclusion in point (ii) follows from [11, Lemma 9.2]1. For the first one, it is enough to show that there exists a universal constant $\bar{s} \in(0,1)$ such that

$$
\bar{s} Q_{h}^{\bar{u}}(\bar{q}) \subset Q_{h / 2}^{\bar{u}}(\bar{q})
$$

and then iterate this estimate (here $\bar{u}$ is defined as in (2.1), and $\bar{q}:=q(x)$ ). To prove (2.19), let $E_{2 h}$ be the John ellipsoid associated to $Q_{2 h}^{\bar{u}}(\bar{q})$, and assume without loss of generality that that $E_{2 h}$ is centered at the origin. By convexity of the sections in this new variables,

$$
\bar{s}\left(Q_{h}^{\bar{u}}(\bar{q})-\bar{q}\right)+\bar{q} \subset Q_{h}^{\bar{u}}(\bar{q}) \subset Q_{2 h}^{\bar{u}}(\bar{q}) \subset n E_{2 h} \quad \forall \bar{s} \in(0,1) .
$$

Observe now that, for any $q \in Q_{h}^{\bar{u}}(\bar{q})$, we have (recall that $\bar{u}(\bar{q})=0$ )

$$
\bar{u}(\bar{s}(q-\bar{q})+\bar{q})=\bar{s} \int_{0}^{1} D \bar{u}((1-t \bar{s}) \bar{q}+t \bar{s} q) \cdot(q-\bar{q}) d t .
$$

Since $q, \bar{q} \in n E_{2 h}$ we have $q-\bar{q} \in 2 n E_{2 h}$, hence

$$
(q-\bar{q}) / 2 n \in E_{2 h} \subset Q_{2 h}(\bar{q}) .
$$

Moreover, by convexity of $Q_{2 h}(\bar{q}),(1-t \bar{s}) \bar{q}+t \bar{s} q \in Q_{h}(\bar{q}) \subset \tau_{0} Q_{2 h}(\bar{q})$ for some universal $\tau_{0}<1$ (see [11, Lemma 9.2]). Defining the "dual norm" $\|\cdot\|_{\mathcal{K}}^{*}$ associated to a convex set $\mathcal{K}$ as

$$
\|a\|_{\mathcal{K}}^{*}:=\sup _{\xi \in \mathcal{K}} a \cdot \xi
$$

it follows from [11, Lemma 6.3] that

$$
\|D \bar{u}(q)\|_{Q_{2 h}(\bar{q})}^{*}=\left\|-D_{q} \bar{c}\left(q, T_{\bar{u}}(q)\right)\right\|_{Q_{2 h}^{\bar{u}}(\bar{q})}^{*} \leq C h \quad \forall q \in Q_{h}^{\bar{u}}(\bar{q}) .
$$

\footnotetext{
${ }^{1}$ To be precise, in 11 the dilation is done with respect to the center of the John ellipsoid, and not with respect to the "center" $x$ of the section. However, it is easy to see that the same statement holds also in this case.
} 
Thus, thanks to (2.20) and (2.21) we get

$$
\begin{aligned}
\bar{u}(\bar{s}(q-\bar{q})+\bar{q}) & =2 n \bar{s} \int_{0}^{1} D \bar{u}((1-t \bar{s}) \bar{q}+t \bar{s} q) \cdot \frac{(q-\bar{q})}{2 n} d t \\
& \left.\leq 2 n \bar{s} \int_{0}^{1} \| D \bar{u}((1-t \bar{s}) \bar{q}+t \bar{s} q)\right) \|_{Q_{2 h}(\bar{q})}^{*} d t \leq 2 n \bar{s} C h \leq h / 2,
\end{aligned}
$$

provided $\bar{s}$ is small enough. This proves the desired inclusion.

As shown for instance in [10], an easy consequence of property (iii) is the following Vitali-type covering theorem.

Proposition 2.3 (Vitali covering theorem). Let $u, f, \Omega^{\prime}, \Omega^{\prime \prime}, \rho, \sigma$ be as in Proposition (2.2), let $D$ be a compact subset of $\Omega^{\prime}$, and let $\left\{S_{h_{x}}(x)\right\}_{x \in D}$ be a family of sections with $h_{x} \leq \rho$. Then we can find a finite number of these sections $\left\{S_{h_{x_{i}}}\left(x_{i}\right)\right\}_{i=1, \ldots, m}$ such that

$$
D \subset \bigcup_{i=1}^{m} S_{h_{x_{i}}}\left(x_{i}\right), \quad \text { with }\left\{S_{\sigma h_{x_{i}}}\left(x_{i}\right)\right\}_{i=1, \ldots, m} \text { disjoint. }
$$

We now want to show that sections at the same height have a comparable shape. For this, we first recall the following estimate from [11]:

Proposition 2.4 (Aleksandrov estimates). Let $u, f, \Omega^{\prime}, \Omega^{\prime \prime}, \rho$ be be as in Proposition (2.2), and let $S_{h}(x)$ be a section of $u$ for some $x \in \Omega^{\prime}$ and $h \leq \rho$. Then

$$
\left|S_{h}\left(x_{0}\right)\right| \approx h^{n / 2} \text {. }
$$

Remark 2.5. Estimates (2.22) and (2.23) have the following important consequence: consider the function $\bar{u}$ defined in (2.1), fix one of its sections $Q_{h}$ such that $Q_{2 h} \subset \Omega^{\prime \prime}$ with $\Omega^{\prime \prime}$ as above, normalize $Q_{h}$ using its corresponding John's transformation $L$, and define $\bar{w}$ as in (2.10). Since $(\operatorname{det} L)^{-2 / n} \approx\left|E_{h}\right| \approx \operatorname{osc}_{Q_{h}} \bar{u} \approx \operatorname{osc}_{Q_{2 h}} \bar{u}$ (by (2.23) $)$ and $E_{h} \subset Q_{2 h}$, we deduce the universal gradient bound

$$
\begin{aligned}
\sup _{L\left(Q_{h}\right)}|D \bar{w}| & =(\operatorname{det} L)^{2 / n} \sup _{Q_{h}}\left|\left(L^{*}\right)^{-1} D \bar{u}\right| \\
& \leq C(\operatorname{det} L)^{2 / n} \sup _{Q_{h}}\|D \bar{u}\|_{E_{h}}^{*} \\
& \leq C(\operatorname{det} L)^{2 / n} \sup _{Q_{h}}\|D \bar{u}\|_{Q_{2 h}}^{*} \\
& \leq C(\operatorname{det} L)^{2 / n} \underset{Q_{2 h}}{\operatorname{osc}} \bar{u} \leq C .
\end{aligned}
$$

Lemma 2.6. Let $u, f, \Omega^{\prime}, \Omega^{\prime \prime}, \rho$ be as in Proposition (2.2). Then for any $0 \leq h \leq \rho$ there exist two radii $r=r(h)$ and $R=R(h)$ such that, for every $x_{0} \in \Omega^{\prime}$, if $E$ is the John ellipsoid associated to $S_{h}^{u}\left(x_{0}\right)$, then, up to a translation,

$$
B_{r}(0) \subset E \subset B_{R}(0) .
$$

Proof. Let $r_{1} \leq \ldots \leq r_{n}$ be the axes of $E$. Since $r_{n} \leq \operatorname{diam}(E) \leq C$ and by (2.23)

$$
h^{n / 2} \approx|E| \approx r_{1} \cdot \ldots \cdot r_{n} \leq \operatorname{diam}(E)^{n-1} r_{1},
$$

we obtain the desired lower bound on $r_{1}$.

Obviously analogous properties holds for the section $Q_{h}^{\bar{u}}\left(q_{0}\right)$. 
Remark 2.7. Notice that Proposition [2.2(ii) applied to the (convex) sections of $\bar{u}$ implies the following: given $x \in \Omega^{\prime \prime}$ and $h \leq \rho$, let $r_{1} \leq \ldots \leq r_{n}$ denote the axes of the John ellipsoid associated to $Q_{h}(x)$. Then

$$
r_{n} \leq C r_{1}^{\alpha_{3}}
$$

for some universal exponent $\alpha_{3}<1$ and a constant $C\left(\Omega^{\prime}, \Omega^{\prime \prime}\right)$.

To see this just normalize $Q_{\rho}(x)$ using $L$ and notice that, by [11, Theorem 6.11] $\operatorname{dist}\left(x, \partial\left(L\left(Q_{\rho}(x)\right)\right) \geq\right.$ $1 / C$ for some universal constant $C$. Thus, up to enlarge $C$,

$$
\left(\frac{h}{C \rho}\right)^{\alpha_{2}} B_{1}(x) \subset L\left(Q_{h}\right) \subset\left(\frac{C h}{\rho}\right)^{\alpha_{1}} B_{1}(x) .
$$

Since, by Lemma 2.6, sections of height $\rho$ have bounded eccentricity (i.e., $|L| \approx C\left(\Omega^{\prime}, \Omega^{\prime \prime}\right)$ ), this implies the claim with $\alpha_{3}:=\alpha_{2} / \alpha_{1}$.

We now observe that $\boldsymbol{\alpha}\left(Q_{h}\right) \approx r_{n}^{2} /\left(r_{1} \ldots r_{n}\right)^{2 / n}$, from which we deduce that

$$
r_{1}^{2} \leq C \frac{r_{n}^{2}}{\boldsymbol{\alpha}\left(Q_{h}\right)}
$$

In particular, this and (2.25) imply

$$
r_{n} \leq C r_{1}^{\alpha_{3}} \leq C \frac{r_{n}^{\alpha_{3}}}{\boldsymbol{\alpha}\left(Q_{h}\right)^{\alpha_{3} / 2}}
$$

that is

$$
r_{n} \leq \frac{C}{\boldsymbol{\alpha}\left(S_{h}\right)^{\beta}}, \quad \text { with } \quad \beta:=\frac{\alpha_{3}}{2-2 \alpha_{3}} .
$$

Hence, since $S_{h}$ is linked to $Q_{h}$ by a diffeomorphism with universal $C^{1}$ norm, and $\operatorname{diam}\left(S_{h}\right) \leq$ $\operatorname{diam}\left(n E_{h}\right)=2 n r_{n}$, we get

$$
\operatorname{diam}\left(S_{h}\right) \leq \frac{\bar{C}}{\boldsymbol{\alpha}\left(S_{h}\right)^{\beta}}, \quad \beta, \bar{C}>0 \text { universal. }
$$

\section{3. $W^{2,1+\kappa}$ ESTIMATES}

Applying first a large dilation to $\Omega$ we can assume that $B(0,1) \subset \Omega$, and by a standard covering argument (see for instance [9, Section 3]) it suffices to prove the $W^{2,1+\kappa}$ regularity of $u$ inside $B(0,1 / 2)$. Also, by an approximation argument 2 , it is enough to prove the result when $u \in C^{2}$. Hence Theorem 1.1 is a consequence of the following:

Theorem 3.1. Let $u \in C^{2}$ be a c-convex solution of (1.4) with $\Omega \supset B(0,1)$. Then there exist universal constants $\kappa$ and $C$ such that

$$
\int_{B(0,1 / 2)}\left|D^{2} u\right|^{1+\kappa} \leq C
$$

We start with the following lemma:

\footnotetext{
${ }^{2}$ To approximate our solution with smooth ones, it suffices to regularize the data and then: - either apply [18, Remark 4.1] (notice that, by Proposition 2.2(iv) and 11, Theorem 8.2], $u$ is strictly $c$-convex and of class $C^{1}$ inside $\Omega$ );

- or approximate our cost $c$ with cost functions satisfying the strong version of (C1) and apply [18, Theorem 1.1].
} 
Lemma 3.2. Let $u$ be as above, $x_{0} \in B(0,3 / 4)$, and $h>0$ such that $S_{2 h}\left(x_{0}\right) \subset B(0,5 / 6)$. Consider the function $\bar{u}$ as in (2.1), its section $Q_{h}=Q_{h}\left(q_{0}\right)$ with $q_{0}:=q\left(x_{0}\right)$, and (up to a rotation) let $E_{h}=\left\{\sum x_{i}^{2} / r_{i}^{2} \leq 1\right\}$ be the John ellipsoid associated to $Q_{h}\left(q_{0}\right)$. Denote by $L$ be the affine transformation that normalizes $Q_{h}$, and define $\bar{w}$ and $\mathcal{C}_{i j, k \ell}$ as in (2.10) and (2.13) respectively. Then

$$
\int_{L\left(Q_{h}\right)}\left|\partial_{i j} \bar{w}-\mathcal{C}_{i j, k \ell} \partial_{k} \bar{w} \partial_{\ell} \bar{w}\right| \leq C
$$

for some universal constant $C$.

Proof. Since, by the $c$-convexity of $u$ (which is preserved under change of variables), the matrix $\left(\partial_{i j} \bar{w}-\mathcal{C}_{i j, k \ell} \partial_{k} \bar{w} \partial_{\ell} \bar{w}\right)_{i, j=1, \ldots, n}$ is non-negative definite, it is enough to estimate

$$
\int_{L\left(Q_{h}\right)} \sum_{i=1}^{n}\left(\partial_{i i} \bar{w}-C_{i i, s t} \partial_{k} \bar{w} \partial_{l} \bar{w}\right)
$$

from above.

Using the bounds $\mathcal{H}^{n-1}\left(\partial\left(L\left(Q_{h}\right)\right)\right) \leq C(n)$ ( since $L\left(Q_{h}\right)$ is a normalized convex set) and $|D \bar{w}| \leq$ $C$ (see (2.24) $)$, we see that first term is controlled from above by

$$
\int_{L\left(Q_{h}\right)} \Delta \bar{w}=\int_{\partial\left(L\left(Q_{h}\right)\right)} D \bar{w} \cdot \nu \leq \mathcal{H}^{n-1}\left(\partial\left(L\left(Q_{h}\right)\right)\right) \sup _{L\left(Q_{h}\right)}|D \bar{w}| \leq C .
$$

For the second term, we claim the following: there exists a universal constant $C$ such that

$$
\inf _{L\left(Q_{h}\right)} \sum_{i=1}^{n} \mathcal{C}_{i i, k \ell} \partial_{k} \bar{w} \partial_{\ell} \bar{w} \geq-C
$$

To see this we write

$$
\sum_{i=1}^{n} \mathcal{C}_{i i, k \ell} \partial_{k} \bar{w} \partial_{\ell} \bar{w}=\sum_{i=1}^{n} \sum_{k, \ell \neq i} \mathcal{C}_{i i, k \ell} \partial_{k} \bar{w} \partial_{\ell} \bar{w}+2 \sum_{i=1}^{n} \sum_{k \neq i} \mathcal{C}_{i i, i k} \partial_{i} \bar{w} \partial_{k} \bar{w}+\sum_{i=1}^{n} \mathcal{C}_{i i, i i} \partial_{i} \bar{w} \partial_{i} \bar{w}
$$

We first observe that, since for any $i=1, \ldots, n$ the vector $\left(\partial_{1} \bar{w}, \ldots, \partial_{i-1} \bar{w}, 0, \partial_{i+1} \bar{w}, \ldots, \partial_{n} \bar{w}\right)$ is orthogonal to coordinate vector $e_{i}$, the first term in the right hand side is non-negative by condition (C1).

Concerning the second and the third term, taking into account the definition of $\mathcal{C}_{i j, k \ell}$ in (2.12) we can rewrite them as

$$
2 \sum_{i=1}^{n} \sum_{k \neq i}\left(r_{1} \ldots r_{n}\right)^{2 / n} \frac{r_{i}}{r_{k}} \mathcal{B}_{i i, i k} \partial_{i} \bar{w} \partial_{k} \bar{w}+\sum_{i=1}^{n}\left(r_{1} \ldots r_{n}\right)^{2 / n} \mathcal{B}_{i i, i i} \partial_{i} \bar{w} \partial_{i} \bar{w} .
$$

Observe that, by (2.23),

$$
\left(r_{1} \ldots r_{n}\right)^{2 / n} \approx h
$$

In addition, by the Lipschitz regularity of $\bar{u}$ (which is simply a consequence of the fact that $u$ is locally Lipschitz inside $\Omega$ ),

$$
h / r_{k} \leq C \quad \forall k=1, \ldots, n .
$$

Since $\|D \bar{w}\|_{\infty} \leq C$ (see (2.24) $)$ and the size of $\mathcal{B}$ is controlled by $\|\bar{c}\| \approx\|c\|$, by (3.6) and (3.7) we see that the expression in (3.5) is universally bounded.

This proves (3.4), which combined with (3.3) concludes the proof. 
Lemma 3.3. With the same notation and hypotheses as in Lemma [3.2, let $\sigma$ be as in Proposition 2.3. Then there exists a universal constant $C$ such that

$$
\left|\left\{\tilde{q} \in L\left(Q_{\sigma h}\right): \operatorname{Id} / C \leq \partial_{i j} \bar{w}-\mathcal{C}_{i j, k \ell} \partial_{k} \bar{w} \partial_{\ell} \bar{w} \leq C \operatorname{Id}\right\}\right| \geq \frac{1}{C} .
$$

Proof. Since $\sigma$ is universal and $L\left(Q_{h}\right)$ is normalized, by Proposition 2.2 (ii) we get

$$
\left|L\left(Q_{\sigma h}\right)\right| \approx\left|L\left(Q_{h}\right)\right| \approx 1
$$

So, using Lemma 3.2 and Chebychev inequality, we deduce the existence of a universal constant $C$ such that

$$
\left|\left\{\tilde{q} \in L\left(Q_{\sigma h}\right): \partial_{i j} \bar{w}-\mathcal{C}_{i j, k \ell} \partial_{k} \bar{w} \partial_{\ell} \bar{w} \leq C \operatorname{Id}\right\}\right| \geq \frac{1}{C} .
$$

Since by (2.11) the product of the eigenvalues of the matrix $\left(\partial_{i j} \bar{w}-\mathcal{C}_{i j, k \ell} \partial_{k} \bar{w}\right)_{i, j=1, \ldots, n}$ is of order one, whenever the eigenvalues are universally bounded from above, they also have to be universally bounded also from below. Hence, up to enlarging the value of $C$, this proves (3.8).

Remark 3.4. Recalling the definition (2.15) of $\boldsymbol{\alpha}\left(Q_{h}\right)=\boldsymbol{\alpha}\left(S_{h}\right)$, we can rewrite both (3.2) and (3.8) in terms of $\bar{u}$ and $Q_{h}=Q_{h}\left(q_{0}\right)$, obtaining that

$$
\int_{Q_{h}}\left|\partial_{i j} \bar{u}-\mathcal{B}_{i j, k \ell} \partial_{k} \bar{u} \partial_{l} \bar{u}\right| \leq C \boldsymbol{\alpha}\left(Q_{h}\right)\left|\left\{x \in Q_{\sigma h}: \boldsymbol{\alpha}\left(Q_{h}\right) / C \leq\left|\partial_{i j} \bar{u}-\mathcal{B}_{i j, k \ell} \partial_{k} \bar{u} \partial_{l} \bar{u}\right| \leq C \boldsymbol{\alpha}\left(Q_{h}\right)\right\}\right|
$$

(see for instance the proof of [10, Lemma 3.2]). In terms of $u$, this estimate becomes

$$
\int_{S_{h}}\left|D^{2} u-\mathcal{A}(x, D u)\right| \leq C_{0} \boldsymbol{\alpha}\left(S_{h}\right)\left|\left\{x \in S_{\sigma h}: \boldsymbol{\alpha}\left(S_{h}\right) / C_{0} \leq\left|D^{2} u-\mathcal{A}(x, D u)\right| \leq C_{0} \boldsymbol{\alpha}\left(S_{h}\right)\right\}\right|,
$$

where $S_{h}=S_{h}\left(x_{0}\right)$ with $x_{0}$ an arbitrary point inside $B(0,3 / 4)$, and $C_{0}$ is universal.

Proof of Theorem [3.1. Let $M \gg 1$ to be fixed later, set $R_{0}:=3 / 4$, and for all $m \geq 1$ define

$$
R_{m}:=R_{m-1}-\bar{C} M^{-\beta} \text {. }
$$

with $\bar{C}$ and $\beta$ as in (2.26). Let us use denote $B\left(0, R_{m}\right)$ by $B_{R_{m}}$, set

$$
F(x):=\left|D^{2} u(x)-\mathcal{A}(x, D u(x))\right|,
$$

and define

$$
D_{m}:=\left\{x \in B_{R_{m}}: F(x) \geq M^{m}\right\} .
$$

Thanks to Proposition 2.2, there exists $\rho>0$ universal such that $S_{h}(x) \subset B(0,5 / 6)$ for any $x \in B(0,3 / 4)$ and $h \leq 2 \rho$, and by Lemma 2.6 applied with $h=\rho$ we get $\boldsymbol{\alpha}\left(S_{\rho}(x)\right) \approx 1$. In addition, since (C0) implies that $|\mathcal{A}(x, D u)| \leq C_{1}$ inside $B(0,1)$ for some $C_{1}$ universal, we see that ||$D^{2} u|-F| \leq C_{1}$. Hence, using Lemma 2.1, we deduce that if $M \gg M_{1}+C_{1}$ then there exists a small universal constant $\nu>0$ such that

$$
\boldsymbol{\alpha}\left(S_{h}(x)\right) \geq \nu M^{m} \quad \forall x \in D_{m}, h \leq \min \{\bar{h}(x), \rho\}, m \geq 1 .
$$

So, by choosing $M \geq \max \left\{1 / \nu^{4}, M_{1}\right\}$ (so that $\nu M^{m+1} \geq M^{m+1 / 2} / \nu$ ), by continuity we obtain that, for every point in $D_{m+1}$, there exists $h_{x} \in(0, \min \{\bar{h}(x), \rho\})$ such that $\boldsymbol{\alpha}\left(S_{h_{x}}\right) \in$ $\left(\nu M^{m+1 / 2}, M^{m+1 / 2} / \nu\right)$. In particular, by (2.26) we have $\operatorname{diam}\left(S_{h_{x}}\right) \leq \bar{C} M^{-\beta}$, which implies that (recall (3.10) $)$

$$
\bigcup_{x \in D_{m+1}} S_{h_{x}} \subset B\left(0, R_{m+1}+\bar{C} M^{-\beta}\right)=B_{R_{m}} .
$$


According to Proposition 2.3, we can cover $D_{m+1}$ with finitely many sections $\left\{S_{h_{x_{j}}}\right\}_{x_{j} \in D_{m+1}}$ such that $S_{\sigma h_{x_{j}}}$ are disjoint. Then (2.17) and (3.9) imply (recall (3.11))

$$
\begin{aligned}
\int_{D_{m+1}} F \leq \sum_{j} \int_{S_{h_{x_{j}}}} F & \leq \sum_{j} C_{0} \boldsymbol{\alpha}\left(S_{h_{x_{j}}}\right)\left|\left\{x \in S_{\sigma h_{x_{j}}}: \boldsymbol{\alpha}\left(S_{h_{x_{j}}}\right) / C_{0} \leq F \leq C_{0} \boldsymbol{\alpha}\left(S_{h_{x_{j}}}\right)\right\}\right| \\
& \leq \sum_{j} \frac{C_{0}}{\nu} M^{m+1 / 2}\left|\left\{x \in S_{\sigma h_{x_{j}}}: \nu M^{m+1 / 2} / C_{0} \leq F \leq C_{0} M^{m+1 / 2} / \nu\right\}\right| .
\end{aligned}
$$

Assuming now that $\sqrt{M} \geq C_{0} / \nu$ and recalling (3.13), we obtain

$$
\begin{aligned}
\int_{D_{m+1}} F \leq \sum_{j} \int_{S_{h_{x_{j}}}} F & \leq \sum_{j} \frac{C_{0}}{\nu} M^{m+1 / 2}\left|\left\{x \in S_{\sigma h_{x_{j}}}: \nu M^{m+1 / 2} / C_{0} \leq F \leq C_{0} M^{m+1 / 2} / \nu\right\}\right| \\
& \leq \sum_{j} \frac{C_{0}}{\nu} M^{m+1 / 2}\left|\left\{x \in S_{\sigma h_{x_{j}}}: M^{m} \leq F \leq M^{m+1}\right\}\right| \\
& =\sum_{j} \frac{C_{0}}{\nu} M^{m+1 / 2}\left|\left\{x \in S_{\sigma h_{x_{j}}}: M^{m} \leq F \leq M^{m+1}\right\} \cap B_{R_{m}}\right| \\
& \leq \frac{C_{0} \sqrt{M}}{\nu} \int_{D_{m} \backslash D_{m+1}} F .
\end{aligned}
$$

Adding $\frac{C_{0} \sqrt{M}}{\nu} \int_{D_{m+1}} F$ to both sides of the previous inequality, we obtain

$$
\left(1+\frac{C_{0} \sqrt{M}}{\nu}\right) \int_{D_{m+1}} F \leq \frac{C_{0} \sqrt{M}}{\nu} \int_{D_{m}} F .
$$

which implies

$$
\int_{D_{m+1}} F \leq(1-\tau) \int_{D_{m}} F
$$

for some small constant $\tau=\tau(M)>0$. We finally fix $M$ so that it also satisfies

$$
\sum_{m \geq 1} \bar{C} M^{-m \beta} \leq \frac{1}{4}
$$

In this way $R_{m} \geq 1 / 2$ for all $m \geq 1$, so that the above inequalities and the definition of $R_{m}$ imply

$$
\int_{\left\{F \geq M^{m}\right\} \cap B(0,1 / 2)} F \leq \int_{D_{m}} F \leq(1-\tau)^{m} \int_{D_{0}} F \leq C(1-\tau)^{m}
$$

(here we used that $\int_{B(0,3 / 4)} F \leq C$, which can be easily proved arguing as in the proof of Lemma 3.2). Thus, choosing $\kappa>0$ such that $1-\tau=M^{-2 \kappa}$, we deduce that

$$
|\{F \geq t\} \cap B(0,1 / 2)| \leq \frac{1}{t} \int_{\{F \geq t\} \cap B(0,1 / 2)} F \leq C t^{-1-2 \kappa}
$$

for some $C>0$ universal, which implies that $F \in L^{1+\kappa}(B(0,1 / 2))$. Recalling the definition of $F$ (see (3.11) ) and that $|\mathcal{A}(x, D u)| \leq C$ inside $B(0,1)$ (by (C0)), this concludes the proof. 


\section{REFERENCES}

[1] L. Ambrosio, M. Colombo, G. De Philippis, A. Figalli: Existence of Eulerian solutions to the semigeostrophic equations in physical space: the 2-dimensional periodic case. Comm. Partial Differential Equations, to appear.

[2] L. Ambrosio, M. Colombo, G. De Philippis, A. Figalli: A global existence result for the semi-geostrophic equations in three dimensional convex domains. Preprint 2012.

[3] L.CAfFarelli: A localization property of viscosity solutions to the Monge-Ampre equation and their strict convexity. Ann. of Math. (2), 131 (1990), no. 1, 129-134.

[4] L.CAffarelli: Some regularity properties of solutions to Monge-Ampère equations. Comm. Pure Appl. Math., 44 (1991), 965-969.

[5] L. Caffarelli: The regularity of mappings with a convex potential. J. Amer. Math. Soc., 5 (1992), 99-104.

[6] M. J. P. Cullen, R. J. Douglas, I. Roulstone, M. J. Sewell: Generalized semi-geostrophic theory on a sphere. J. Fluid Mech., 531 (2005), 123-157.

[7] P. Delanö̈, Y. GE: Regularity of optimal transport on compact, locally nearly spherical, manifolds. J. Reine Angew. Math., 646 (2010), 65-115.

[8] P. Delanoë, Y. Ge: Locally nearly spherical surfaces are almost-positively c-curved. Methods Appl. Anal., 18 (2011), no. 3, 269-302.

[9] G. De Philippis, A. Figalli: $W^{2,1}$ regularity for solutions of the Monge-Ampère equation. Invent. Math., to appear.

[10] G. De Philippis, A. Figalli, O. Savin: A note on interior $W^{2,1+\varepsilon}$ estimates for the Monge-Ampère equation. Math. Ann., to appear.

[11] A. Figalli, Y.H. Kim, R.J. MCCAnN: Hölder continuity and injectivity of optimal maps. Preprint, 2011.

[12] A. Figalli, G. Loeper: $C^{1}$ regularity of solutions of the Monge-Ampère equation for optimal transport in dimension two. Calc. Var. Partial Differential Equations, 35 (2009), no. 4, 537-550.

[13] A. Figalli, L. Rifford: Continuity of optimal transport maps and convexity of injectivity domains on small deformations of $\mathbb{S}^{2}$. Comm. Pure Appl. Math., 62 (2009), no. 12, 1670-1706.

[14] A. Figalli, L. Rifford, C. Villani: Nearly round spheres look convex. Amer. J. Math., 134 (2012), no. 1, 109-139.

[15] F. JoHn: Extremum problems with inequalities as subsidiary conditions. In Studies and Essays Presented to R. Courant on his 60th Birthday, January 8, 1948, pages 187-204. Interscience, New York, 1948.

[16] Y.-H. Kim, R. J. MCCANn: Continuity, curvature, and the general covariance of optimal transportation. J. Eur. Math. Soc. (JEMS), 12 (2010), no. 4, 1009-1040.

[17] J. LıU: Hölder regularity of optimal mappings in optimal transportation. Calc. Var. Partial Differential Equations, 34 (2009), no. 4, 435-451.

[18] J. Liu, N.S. Trudinger, X.-J. WAng: Interior $C^{2, \alpha}$ regularity for potential functions in optimal transportation. Comm. Partial Differential Equations, 35 (2010), 165-184.

[19] G. Loeper: On the regularity of solutions of optimal transportation problems. Acta Math., 202 (2009), no. 2, $241-283$.

[20] G. LOePeR: Regularity of optimal maps on the sphere: The quadratic cost and the reflector antenna. Arch. Ration. Mech. Anal., 199 (2011), no. 1, 269-289.

[21] X. N. Ma, N. S. Trudinger, X. J. WAng: Regularity of potential functions of the optimal transportation problem. Arch. Ration. Mech. Anal., 177 (2005), no. 2, 151-183.

[22] T. Schmidt: $W^{2,1+\varepsilon}$ estimates for the Monge-Ampère equation. Preprint 2012.

[23] N. S. Trudinger, X. J. WAng: On the second boundary value problem for Monge-Ampère type equations and optimal transportation. Ann. Sc. Norm. Super. Pisa Cl. Sci. (5), 8 (2009) 143-174.

[24] N. S. Trudinger, X. J. WANG: On strict convexity and continuous differentiability of potential functions in optimal transportation. Arch. Ration. Mech. Anal., 192 (2009), 403-418.

Scuola Normale Superiore, P.ZA Dei Cavalieri 7, I-56126 Pisa, Italy

E-mail address: guido.dephilippis@sns.it

The University of Texas at Austin, Mathematics Dept. RlM 8.100, 2515 Speedway Stop C1200, Austin, Texas 78712-1202, USA

E-mail address: figalli@math.utexas.edu 